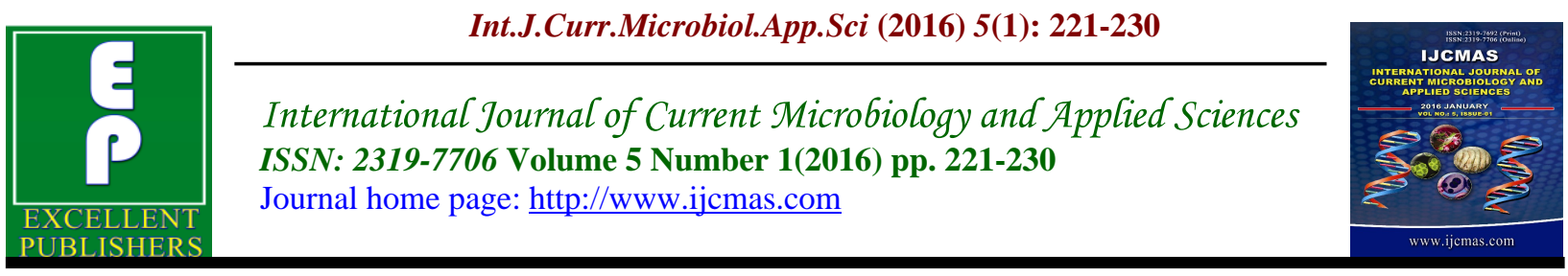

Original Research Article

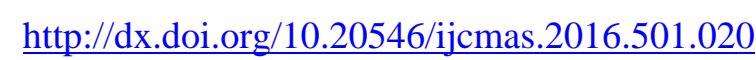

\title{
Down Regulation of MIR-320 Gene Family Members in the Peripheral Blood of Schizophrenia Patients
}

\section{Tihomir Iliev Vachev ${ }^{1,2} *$, Nikolay Todorov Popov ${ }^{4}$, Vili Krasteva Stoyanova ${ }^{2,3}$, Hristo Yordanov Ivanov ${ }^{2}$ and Danail Savov Minchev ${ }^{1}$}

\author{
${ }^{1}$ Department of Plant Phisyology and Molecular Biology, University of Plovdiv "Paisii \\ Hilendarski”, 24 Tzar Assen Str., Plovdiv, Bulgaria \\ ${ }^{2}$ Department of Pediatrics and Medical Genetics, Medical Faculty - Plovdiv, 15A Vassil Aprilov \\ St., 4000 Plovdiv, Bulgaria \\ ${ }^{3}$ University Hospital "St. George” Plovdiv, 66 Peshtersko Shuse Str., Plovdiv 4000, Bulgaria \\ ${ }^{4}$ State Phsychiatry Hospital Pazardzhik, 28 Bolnichna Str. Pazardzhik, Bulgaria
}

*Corresponding author

\begin{tabular}{|c|c|}
\hline & A B S T R A C T \\
\hline & \multirow{13}{*}{$\begin{array}{l}\text { Schizophrenia is a severe disabling brain disease affecting about } 1 \% \text { of the world's } \\
\text { population. Due to the dynamic nature of the peripheral blood transcriptome, } \\
\text { understanding miRNAs gene expression signature in schizophrenia is a promising } \\
\text { tool for discovery of disease-related biomarkers and biological pathways involved. } \\
\text { This study aimed to identify specific miRNA signature characteristic for } \\
\text { schizophrenia by using genome-wide miRNA expression profiling in patients with } \\
\text { schizophrenia vs. healthy controls. In the current study, we examined miRNA gene } \\
\text { expression changes applying custom made LC Science miRNA expression } \\
\text { profiling service, by using pooled whole blood-derived total RNA samples in order } \\
\text { to evaluate possible miRNAs molecules associated with the disease. Here, we } \\
\text { report experimental proof for a differentially down expressed miR-320a, miR- } \\
\text { 320b, miR-320d and miR-320c members. To elucidate the biological pathways } \\
\text { implicated in schizophrenia, additionally we searched for the validated target genes } \\
\text { of miR-320 gene family that might play a role in the regulation of schizophrenia } \\
\text { susceptibility genes using miRWalk database. This analysis revealed } \\
\text { experimentally validated targets with specific function in nervous system and } \\
\text { development including RCOR1, AGO1, REST, and EZH2. Finally, our results } \\
\text { suggested that differentially expressed miR-320 family members might be involved } \\
\text { in schizophrenia molecular pathways. }\end{array}$} \\
\hline Keywords & \\
\hline & \\
\hline 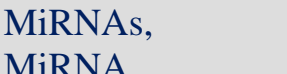 & \\
\hline & \\
\hline & \\
\hline & \\
\hline & \\
\hline & \\
\hline & \\
\hline & \\
\hline & \\
\hline & \\
\hline
\end{tabular}

\section{Introduction}

Schizophrenia (SZ) is a severe mental disorder affecting approximately $1 \%$ of the human population world-wide (Perala et al.,
2007). SZ is a chronic neuropsychiatric disorder characterized by psychosis, emotional dysregulation, 
neuromorphological and cognitive impairment, leading to deteriorated social functioning. Currently, numerous molecular genetic studies, including, micro RNA and protein coding gene expression, candidate gene association analyses, genome-wide association studies (GWAS) and deep sequencing have identified particular genes and chromosomal loci associated with the disease state (Shi at al., 2009; Stefansson at al., 2009; Lai et al., 2011; Simon et al., 2011). MicroRNAs (miRNAs) are evolutionary conserved small noncoding RNAs that have been shown to mediate the posttranscriptional regulation of at least onethird of protein-coding genes (Bartel, 2004; Filipowicz, at al., 2008). In mammals, the greater part of endogenous miRNA genes are transcribed initially as primary transcripts (pri-miRNAs) that range from hundreds to thousands of nucleotides in length and contain one or more extended hairpin structures (Du and Zamore, 2005). MiRNA could also negatively regulate protein expression through targeting of mRNA coding regions (Tay et al., 2008). Furthermore, miRNAs are found to upregulate the translation of target mRNAs in a cell cycle dependent manner, switching between translational suppression in proliferating cells to translational activation in quiescent cells (Vasudevan et al., 2008; Vasudevan 2007a,b). The discovery of endogenous regulatory noncoding RNA, has led to wide spread interest because of the implication to several human diseases, including schizophrenia (Beveridge at al., 2008; Perkins at al., 2007; Beveridge et al., 2009). These molecules strongly influence gene expression, particularly in the brain where they are thought to be involved in both neurodevelopment and in the regulation of synaptic structure and function (Schratt et al., 2006; Sempere et al., 2004). To date, several candidate genes have been examined in order to evaluate their possible associations with the disorder. The examination of posttranscriptional regulation in schizophrenia by evaluating ncRNAs (e.g., miRNAs) can provide a link between PCG regulation of expression and epigenetic factors. Research on gene expression in schizophrenia has previously paying attention on identifying specific or a limited group of genes related to disease (Mari et al., 2013). The idea that alterations at the global level of protein coding gene expression can be postranscriptionaly dysregulated by miRNA-directed transcriptional gene silencing and contribute to schizophrenia risk has been principally underexplored. Peripheral blood is effortlessly accessible material and has been suggested as an alternative to tissue samples for molecular profiling of human disease and assessing disease risk (Zoghbi, 2005). Due to the capacity of whole blood to reflect pathological changes in the body, the identification of miRNAs has significantly expanded the knowledge of the regulatory mechanisms of gene expression. Microarray-based gene expression analysis of peripheral whole blood is a common and versatile strategy in the development of clinically relevant biomarker panels for a variety of human diseases. The human genome contains eight annotated micro RNA 320 genes that encode five mature miRNA variants including miR-320a (Chr 8), miR-320b (Chr 1), miR-320c (Chr 18), miR-320e (Chr 19), miR-320d-1 (Chr 13) and miR-320d-2 (Chr X), with the same mature miRNA sequences. Recently, miR320a was identified as a potential modulator of aquaporin 1 and 4 exploring the possibility of miR-320a to alter the expression of aquaporin 1 and 4 in normal and ischemic conditions (Sugunavathi et al., 2010). The current study aim to shade a light on dysregulation of particular family of miRNAs through microarray technology. 


\section{Materials and Methods}

\section{Participants}

This study design and the Inform Concent Form (ICF) were approved by the Ethics Committee of Plovdiv Medical University. The Institutional Review Board of the university approved the use of the samples for this study. After obtaining written informed consent thirty schizophrenic patients were recruited from State Psychiatric Hospital - Pazardzhik, Bulgaria. Routine psychiatric examination, wide medical history and The Mini-International Neuropsychiatric Interview were done by a certified psychiatrist to evaluate the diagnosis of paranoid SZ only, on Diagnostic and Statistical Manual of Mental Disorders forth text revised edition (DSM IV TR) criteria and in order to exclude any mental disorder in the controls. The sample population included 15 males/15 females diagnosed with SZ and 25 age- and gendermatched general population controls (13 males/12 females) (Table 1.) Main inclusion criteria was that the participants did not receive any medication before blood sampling for at least 2 weeks. Persons with other medical illness were also excluded from the cohorts of interest.

\section{Blood Collection and RNA Extraction}

An aliquot of whole blood (2.5 ml) for each subject (schizophrenia and healthy controls) was collected directly into PAXgene blood RNA tubes (PreAnalytiX) and stored at room temperature for minimum of 4 hours and than freezed at $-20^{\circ} \mathrm{C}$, before extracting total RNA. Total RNA was isolated using PAXgene blood miRNA kit (PreAnalytiX), according to the manufacturer's protocol. Assessment of A260/A280 ratios revealed that all RNA samples are with sufficient quality for microarray analysis $(1.93-2.10)$. RNA quality and purity were analyzed by
Epoch Micro-Volume Spectrophotometer System (BioTek). Two pooled RNA samples (schizophrenia and healthy controls) were created by adding an equivalent amount of total RNA from each individual speciment to final concentration of $5 \mu \mathrm{g}$ total RNA. RNA integrity of pooled samples (schizophrenic and healthy controls) was checked by agarose gel electrophoresis followed by assessment with Agilent 2100 Bioanalyzer.

\section{MicroRNA Expression Analysis (LC Science)}

The $\mu$ Paraflo ${ }^{\mathrm{TM}}$ miRNA microarray assay was performed using a service provider (LC Sciences, Houston, TX) with a proprietary microfluidic array based on the Sanger miRBase _ v18.0 database (http://Www.sanger.ac.uk/Software/Rfam/mi rna). Custom MiRNA microarray analysis was used to find out differential expression of peripheral blood miRNAs between pooled samples of healthy individuals versus pooled RNA samples obtained from schizophrenia patients. The microRNA expression assay required $5 \mu \mathrm{g}$ total RNA sample, which was size fractionated using a YM-100 Microcon centrifugal filter (Millipore, Billerica, MA) and the small RNAs (300 nt) isolated were 3'-extended with a poly (A) tail using poly(A) polymerase. An oligonucleotide tag was then ligated to the poly (A) tail for later fluorescent dye staining. One tag was used for the two RNA samples in one sample experiments. Hybridization was performed overnight on a $\mu$ Paraflo microfluidic chip using a microcirculation pump (Atactic Technologies, Houston, TX). On the microfluidic chip, each detection probe consisted of a chemically modified nucleotide coding segment complementary to target all microRNA and a spacer segment of polyethylene glycol to extend the coding segment away from the substrate. 
Internal control probes were used for quality control of the chip production, sample labeling, and assay conditions. The detection probes were made by in situ synthesis using photogenerated reagentchemistry. The hybridization melting temperatures were balanced by chemical modifications of the detection probes. After RNA hybridization, tag-conjugating Cy5 dye was circulated through the microfluidic chip for dye staining. Fluorescence images were collected using a GenePix 4000B laser scanner (Molecular Device, Sunnyvale, CA) and digitized using Array-Pro image analysis software (Media Cybernetics, Bethesda, MD).

\section{Target Analysis of Schizophrenia- Associated MIR- 320 Members}

Each differentially down expressed miRNA molecule has potential to regulate numerous target genes and therefore can modulate multiple pathways. In order to explore which protein coding gene targets and pathways might be influenced by these differentially expressed miRNA members, we used the miRWalk database as an important resource for the functional investigation of validated and putative individual miRNA targets. Firstly, we identified putative target genes (mRNAs) of these miR-320 family members using the publically available database - miRWalk, available at (http://mirwalk.uni-hd.de/) (Dweep et al., 2011). In addition, the validated targets module was used to give information about experimentally validated miRNAs protein - coding genes interaction.

\section{Statistical Methods}

\section{Microarray Data Analysis}

Data analysis included the determination of detectable signals, calculation of signal intensities, and calculation of differential ratios ( $\log 2$ transformed) of the two technical replicates. The data process began with background subtraction, detectivity determination, and then $P$-value calculation.

\section{Statistical Methods}

To determine differentially expressed miRNAs Welch $\mathrm{T}$ - test adaptation was done, assuming unequal variances. The significant $P$-value used was $<0.05$.

\section{Results and Discussion}

To date, several candidate genes have been examined to evaluate their possible associations with the disease. These candidate genes have generally been selected based on supportive linkage/cytogenetic evidence or the presence of certain findings at the clinical level in subjects with schizophrenia. One of the main techniques in obtaining these candidate genes has been direct sequencing of gene exons using genomic DNA from subjects with schizophrenia compared with control group. The study of post-transcriptional regulation in schizophrenia by evaluating ncRNAs (e.g., miRNAs) can provide a link between disease susceptibility and potential epigenetic factors. Several observations can be used in support of the assumption that microRNAs are molecules that would be rational to test in pathogenesis of brain disorders such as schizophrenia. Some microRNAs and other small RNAs show coexpression in whole blood and brain, there are brain enriched microRNA species also expressed in peripheral whole blood. In this study, we characterized the expression of miR-320 family members in peripheral blood samples of schizophrenia patients. Using $\mu$ Paraflo $^{\mathrm{TM}}$ miRNA microarray assay we found that miR-320 members are differentially expressed (down-regulated) in peripheral blood with statistical significance (miR-320a; $p=0,032)$, (miR-320b; $p=0,034)$, (miR-320c; $\quad p=0,0054), \quad$ (miR-320d; 
$p=0,0314)$ accordingly. The results from the miRNA microarray are depicted in heat maps, in which the expression of each miRNA across samples was assigned a color value (Figure 1).

This strategy offers an opportunity to take the first step in understanding the role of miRNAs and their mRNAs targets in the etiology of schizophrenia. The miR-320 members have many experimentally validated targets as well as several potential targets with a set of interesting neural active functions. Validated target genes of miR320d includes; RBFOX2, (RNA binding protein fox-1 homolog 2) RNA-binding protein that regulates alternative splicing events by binding to 5'-UGCAUGU-3' elements. RBFOX2 regulates alternative splicing of tissue-specific exons and of differentially spliced exons during erythropoiesis. SLC38A1 is an important transporter of glutamine. Glutamine serves as a precursor for the synaptic transmitter, glutamate. Targets of miR-320c includes; REST (E1-silencing transcription factor). This protein is also found in undifferentiated neuronal progenitor cells and it is thought that this repressor may act as a master negative regulator of neurogenesis (Rosalba D A., and Jacopo M. 2013). AGO1, mentioned earlier, with his role in RNA interference; RCOR1 (REST corepressor 1) - gene encodes a protein that is wellconserved, downregulated at birth, and with a specific role in determining neural cell differentiation. The encoded protein binds to the C-terminal domain of REST (repressor element-1 silencing transcription factor) (citat). Specific validated targets of other family member miR-320b includes: EZH2 (enhancer of zeste homolog 2), protein that may play a role in the hematopoietic and central nervous systems. And a REST corepressor 1 which is common target with miR-320a. A list of validated target genes are shown in Table 2.

Table.1 Demographic Information for the Schizophrenic and Control Cohorts

\begin{tabular}{lll}
\hline \multicolumn{1}{c}{ Demographic } & \multicolumn{1}{c}{ Schizophrenia $(\mathrm{n}=30)$} & \multicolumn{1}{c}{ Control $(\mathrm{n}=25)$} \\
\hline Sex, M/F & 15 male; 15 female & 13 male; 12 female \\
Mean age/years & 45.62 & 46.38 \\
Age range/years & & \\
\hline
\end{tabular}

Figure.1 Heat Map Displaying MIR-320b, d, c, and MIR-320a Expression in Whole Blood Samples from (SZ) and Healthy Controls (SZC) by MicroRNA Microarray Assay. Increased Expression are shown in Red, Decreased - in Green; No/Little Changes - in Black

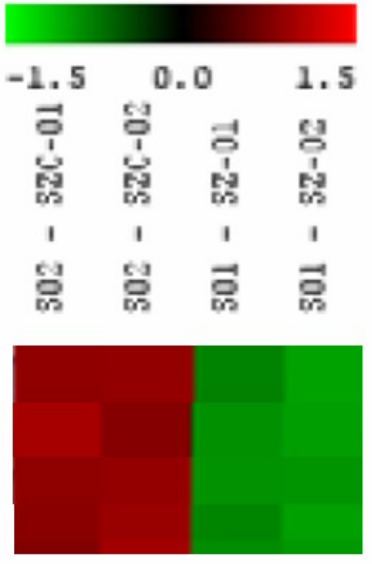

MiR-320a

MiR-320b

MiR-320c

MiR-320d 
Table.2 List of Validated Target Genes of Dysregylated MIRNAs

\begin{tabular}{|c|c|c|}
\hline MiRNAs & Dysregulation & Validated target genes \\
\hline miR-320a & Downregulated & $\begin{array}{l}\text { ACTB, ACTG1, ACTN4, ADCY1, AK2, AMELX, ANXA11, } \\
\text { AQP1, AQP4, ARF3, RERE, ATP1A1, ATP5B, ATP6V1B2, } \\
\text { ATP7A, AUP1, BYSL, CALM3, CALR, CALU, CANX, CAPNS1, } \\
\text { CCND2, CD44, CDK4, CDKN2A, CKB, COX6B1, CPD, CSE1L, } \\
\text { CTNS, CTPS1, CYBA, CYLD, DIAPH1, SEPT1, DPYSL2, DR1, } \\
\text { DUSP8, DVL3, ECH1, EEF2, EIF4G1, MARK2, ENPEP, EPB41, } \\
\text { EPHA4, EWSR1, EXT1, EZH2, FHL3, FKBP1A, FMO1, GAPDH, } \\
\text { GART, GHSR, GLUL, GNAS, GRK6, HIST1H1E, H2AFX, } \\
\text { HIST1H1A, HSD17B10, HOXA10, HOXB8, HSPA4, HSPA8, } \\
\text { HSPA9, HSP90AB1, IDH3G, IGF1R, ILF3, KPNB1, LAMA5, } \\
\text { ABLIM1, LMNB1, MAN2A1, MCL1, MCM4, DNAJB9, MDH2, } \\
\text { MEF2D, MEOX2, MET, MIF, KMT2A, MOCS1, MPI, MYC, } \\
\text { MYO1D, MYO6, NDUFA3, NEO1, TONSL, NQO2, NPM1, NPR1, } \\
\text { NRF1, OAZ1, OCRL, OPA1, ORC1, P4HA1, PAK1, PCBP2, } \\
\text { PFKM, PITX1, PKM, PLS1, PLS3, PNN, POLR2A, POLR2F, } \\
\text { PPIA, PPP1R7, PPP4C, PPT1, MAPK1, PSMD10, PSMD11, } \\
\text { PTGS1, PTGS2, PTPN9, PTPN11, PVRL2, RAB2A, RAD23A, } \\
\text { RAN, RELA, RPL7A, RPL8, RPL9, RPL27, RPL30, RPLP1, } \\
\text { RPS4X, RPS6KA3, RPS12, RPS16, RPS17, RPS27, SALL1, SBF1, } \\
\text { ATXN1, SDHC, SEC14L1, SH3GL1, SLC2A1, SLC6A8, } \\
\text { SLC16A1, SMARCC2, SNRPB, SNRPD2, SNRPG, CAPN15, SRF, } \\
\text { SRM, SRP68, SUPV3L1, TAC1, HNF1A, TCF3, MLX, TCP1, } \\
\text { TEAD1, TFAP2A, TFRC, TFRC, TFRC, TMPO, TOP3A, TPI, } \\
\text { CCT3, TSC1, TUB, UBA52, UBE2G1, USF2, VAV2, VCL, } \\
\text { VDAC1, VDAC2, WARS, XBP1, YWHAE, SF1, ZFP37, ZIC2, } \\
\text { ZNF155, ZFAND5, CSDE1, RNF103, ABHD16A, PDHX, } \\
\text { PTP4A2, KMT2D, ANP32A, TAF15, KDM5C, SMC1A, AXIN1, } \\
\text { HIST2H2AA3, HIST1H2BL, HIST1H2BH, HIST1H2BC, } \\
\text { HIST1H2BO, HIST1H3H, HIST1H3B, PIP4K2B, ULK1, ZNF282, } \\
\text { EIF3C, EIF3F, EIF3J, CTNNAL1, CDS2, EIF2B3, BTRC, } \\
\text { HIST1H2BJ, CLDN12, PDLIM1, SYNGR2, DYRK1B, ARHGEF2, } \\
\text { PTTG1, CYTH3, GTF3C4, MED23, MED26, ITM2B, PSMF1, } \\
\text { LITAF, NPEPPS, CHD1L, PRDX6, NCOR1, TRAF4, ZNF432, } \\
\text { KIAA0100, NOS1AP, ZNF646, SETD1A, TSC22D2, TRIM14, } \\
\text { ZEB2, EPM2AIP1, MED24, POM121, UBAP2L, DENND4B, } \\
\text { RNF10, MED12, ABCF2, DPP3, HUWE1, PPIF, G3BP1, RNF41, } \\
\text { USPL1, FLOT1, SPRY3, CDK2AP2, NET1, TUBA1B, PRMT5, } \\
\text { MCRS1, COG5, PFDN6, SSSCA1, ANP32B, SORBS1, USP16, } \\
\text { PAICS, IVNS1ABP, IGF2BP1, IGF2BP3, TOB2, ARPP19, } \\
\text { CPEB3, RALY, SPEN, ERC1, ARHGAP26, TNRC6B, MAPK8IP3, }\end{array}$ \\
\hline
\end{tabular}




\begin{tabular}{|c|c|c|}
\hline & & $\begin{array}{l}\text { ZC3H4, XPO6, PRRC2C, RPRD2, CLUH, NCAPD3, SATB2, } \\
\text { USP22, KIAA0368, FRAT2, COTL1, SF3B3, CBX5, BRD4, } \\
\text { R3HDM1,RPL13A, CABIN1, SRRM2, SMUG1, PDSS1, PLD3, } \\
\text { ZNF318, DNAJB5, METTL7A, RPL36, SYF2, GORASP2, RPAP1, } \\
\text { TMEM98, FAM127B, GGA1, SENP3, AGO1, RANBP6, FOXP1, } \\
\text { TNRC6A, NXT1, REPIN1, NRBP1, NT5C, STOML2, PIGP, } \\
\text { ANKRD39, NT5C3A, BET1L, GOLM1, EIF3L, ISYNA1, PPIL1, } \\
\text { SIX4, RNF216, PAF1, HAUS6, WBP1L, BCOR, ANKHD1, } \\
\text { INO80D, COMMD4, RNF125, CPSF3L, C20orf27, CLN6, } \\
\text { TMEM51, SAMD4B, ARHGAP17, SYNJ2BP, TMCO6, HES6, } \\
\text { RBM38, NPLOC4, PACS1, POLR3E, ATF7IP, ZFP64, TDP1, } \\
\text { RNF130, UBAP2, CMAS, NXT2, MYO5C, NSFL1C, C20orf24, } \\
\text { CTNNBL1, CDC42SE1, UBQLN4, SMARCAD1, NIT2, RTN4, } \\
\text { PRR12, TBC1D14, MARCH4, EP400, ZNF462, SCAF1, KMT2C, } \\
\text { FAM60A, TSPYL2, WDR13, METTL17, DCLRE1B, RFX7, } \\
\text { MRPS5, MRPL9, INTS3, WNK1, VKORC1, NOC4L, WDR77, } \\
\text { PANK3, YRDC, FBXO31, NANOG, DOK3, EDC3, PROSER1, } \\
\text { ORAI2, FLAD1, DCAF11, ZNF436, VOPP1, CDCA3, GRWD1, } \\
\text { LONP2, TMTC1, ITFG3, USP42, ASCC2, SETD3, UQCC2, } \\
\text { TMEM101, GPT2, PSRC1, ZNF607, ZNF496, LRCH3, DIRC2, } \\
\text { C12orf52, ALKBH6, HIST1H2BK, PPIL4, EAF1, DNAJC14, } \\
\text { UNC5A, PYGO2, ATPAF2, IMP4, TJAP1, PPP1R14A, PLCD3, } \\
\text { TOE1, IKBIP, EARS2, CANT1, LSM12, HSPB6, IFFO2, ARL9, } \\
\text { SNRNP48, KCTD7, ZNF600, DCP2, TMEM64, DTX3, UNC13D, } \\
\text { TUBB, DHRSX, OTUD1, PRR14L, MMS22L, ANKRD52, } \\
\text { SPRYD4, HIST2H3A, IRF2BP2, ZNF710, YPEL2, FAM102A, } \\
\text { ATXN7L3B, HIST2H4B, PPIAL4G, FAM83G }\end{array}$ \\
\hline & $\mathrm{D}$ & $\begin{array}{l}\text { ACLY, ARF1, ATP5B, COX6B1, DNASE2, ATN1, EPB41, GLUL, } \\
\text { GNAS, HOXC11, MKLN1, KMT2A, MYC, PPIA, MAPK3, PURB, } \\
\text { RTKN, SH3GL1, SRP68, TCEB3, MLX, TPI1, CCT3, UBA52, } \\
\text { UQCRC1, VCL, RNF103, PTP4A2, TIMELESS, HIST1H2BJ, } \\
\text { PLOD3 } \\
\text { EPM2AIP1, SLC23A2, G3BP1, RBM5, IVNS1ABP, EHMT2, } \\
\text { HNRNPUL1, ZNF507, PDCD11, ERC1, SATB2, TRPC4AP, } \\
\text { CHCHD2, ZNF581, MAP7D1, ATF7IP, CMAS, PHF12, LY6G5B, } \\
\text { GOLPH3, DCLRE1B, TANGO6, VOPP1, ITFG3, C12orf52, } \\
\text { ZNF600, TXLNA, TUBB, TMEM256, BCL9L, HIST2H3A }\end{array}$ \\
\hline$-320 \mathrm{c}$ & Downregulated & $\begin{array}{l}\text { RHOA, LDHA, PTGS1, RARG, SEC14L1, SNRPA, TSPYL1, } \\
\text { VDAC1, AXIN1, HIST2H2AA3, EIF3C, DYRK1B, SCRN1, } \\
\text { ATG7, IVNS1ABP, LSM14A, AGO1, ITSN2, CYCS, PCDHA2, } \\
\text { EDC3, TTL, TUBB, ATXN7L3B }\end{array}$ \\
\hline miR-320d & Downregulated & RBFOX2, SLC38A1 \\
\hline
\end{tabular}


During the past decade, global gene expression profiling has become increasingly and more reliably used to obtain disease-specific information from blood samples. Despite the accumulating evidence linking miRNAs to various diseases, very little is known about how these small RNAs contribute to the disease. Moreover, we have demonstrated that whole blood microRNA 320 levels could reflect pathophysiological conditions, such as schizophrenia.

The results of this study clearly support our basic hypothesis that microRNAs signature in the whole blood can serve as a promising candidate biomarkers. Furthermore, we used ROC analyses of microRNA signature of these four microRNAs in whole blood to test the diagnostic value as prediction model. The results showed that all of these four microRNAs could serve as valuable biomarkers for schizophrenia. Overall, our study suggests that evaluation of miRNA expression profiling may have potential to identify biological pathways involving protein-coding genes, implicated in schizophrenia. Nevertheless, further studies are necessary to better elucidate the functions and mechanisms involved of the relevant microRNAs 320 family in schizophrenia. Current study outlines that altered miRNAs expression levels are observed in peripheral whole blood from schizophrenia patients, a findings which suggests that dysregulation of miRNAs may contribute to schizophrenia phenotype. Further molecular analysis on miRNA gene expression changes will give a more detailed view about the miRNA associated mechanism in schizophrenia. In the near future, the observed miRNAs expression changes may be able to help define the etiology, genetics and clinical phenotype in schizophrenia.

\section{Acknowledgement}

The authors are most grateful to the probands for its collaboration. This study was supported by MU - Plovdiv Project № HO $\square$ 21/2014.

\section{References}

Bartel, DP. 2004. MicroRNAs: genomics, biogenesis, mechanism, and function. Cell 116:281-297.

Beveridge, N.J., Gardiner, E., Carroll, A.P., Tooney, P.A., Cairns, M.J. 2010. Schizophrenia is associated with an increase in cortical microRNA biogenesis. Mol. Psychiatry 15:11761189.

Beveridge, N.J., Tooney, P.A., Carroll, A.P., Gardiner, E., Bowden, N., Scott, R.J., Tran. N., Dedova, I., Cairns, M.J. 2008. Dysregulation of miRNA $181 \mathrm{~b}$ in the temporal cortex in schizophrenia. Hum. Mol. Genet. 17:1156-1168.

Bingham, A.J., Ooi, L., Kozera, L., White, E., Wood, I.C. 2007. The Repressor Element 1-Silencing Transcription Factor Regulates Heart-Specific Gene Expression Using Multiple Chromatin-Modifying Complexes. Mol. Cell. Biol._27(11):4082-4092

D'Alessandro, R., Meldolesi, J. 2013. Expression and function of the densecore vesicle membranes are governed by the transcription repressor REST. Febs Leters 13:1915-1922

Du, T. and Zamore, P.D. 2005. microPrimer: the biogenesis and function of microRNA. Development 132:46454652.

Filipowicz, W., Bhattacharyya, SN and Sonenberg N. 2008. Mechanisms of post-transcriptional regulation by microRNAs: are the answers in sight? Nat. Rev. Genet 9, 102-114. 
Girard, S.L., Gauthier, J., Noreau, A., Xiong, L., Zhou, S., Jouan, L., Dionne-Laporte, A., Spiegelman, D., Henrion, E., Diallo, O.,Thibodeau, P., Bachand, I., Bao, J.Y., Tong, A.H., Lin, C.H., Millet, B., Jaafari, N., Joober, R., Dion, P.A., Lok, S., Krebs, M.O., Rouleau, G.A. 2011. Increased exonic de novo mutation rate in individuals with schizophrenia. Nat. Genet 43,860-863.

Lai, C.Y., Yu, S.L., Hsieh, M.H., Chen, C.H., Chen, H.Y., Wen, C.C., Huang, Y.H., Hsiao, P.C., Hsiao, C.K., Liu, C.M., Yang, P.C.,, Hwu H.G., Chen, W.J. 2011. MicroRNA expression aberration as potential peripheral blood biomarkers for schizophrenia. PLoS One 6:e216-35.

Perälä, J., Suvisaari, J., Saarni, S.I., Kuoppasalmi, K., Isometsä, E., Pirkola, S., Partonen, T., TuulioHenriksson, A., Hintikka, J., Kieseppä, T., Härkänen, T., Koskinen, S., Lönnqvist, J., 2007. Lifetime prevalence of psychotic and bipolar I disorders in a general population. Arch. Gen. Psychiatry 64:19-28.

Perkins, D.O., Jeffries. C.D., Jarskog,. L.F, Thomson, J.M., Woods, K., Newman, M.A., Parker, J.S., Jin, J., Hammond, S.M. 2007. MicroRNA expression in the prefrontal cortex of individuals with schizophrenia and schizoaffective disorder. Genome Biol. 8:R27.

Rossi, M., Kilpinen, H., Muona, M., Surakka, I., Ingle, C., Lahtinen, J., Hennah, W., Ripatti, S., Hovatta, I. 2013. Allele-specific regulation of DISC1 expression by miR-135b-5 Eur. J. Hum. Genet 1-4

Schratt, G.M., Tuebing, F., Nigh, E.A., Kane, C.G., Sabatini, M.E., Kiebler, M., Greenberg, M.E. 2006. A brainspecific microRNA regulates dendritic spine development. Nature 439:283-
289.

Sempere, L.F., Freemantle, S., Pitha-Rowe, I., Moss, E., Dmitrovsky, E., Ambros, V. 2004. Expression profiling of mammalian microRNAs uncovers a subset of brain-expressed microRNAs with possible roles in murine and human neuronal differentiation. Genome Biol.:5: R13.

Sepramaniam, S., Armugam, A., Lim, K.Y., Karolina, D.S., Swaminathan, P., Tan, J.R., Jeyaseelan, K. 2010. MicroRNA 320a Functions as a Novel Endogenous Modulator of Aquaporins 1 and 4 as Well as a Potential Therapeutic Target in Cerebral Ischemia. J. Biol. Chem. 38: 2922329230.

Shi, J., Levinson, D.F., Duan, J., Sanders, A.R., Zheng, Y., Pe'er, I., Dudbridge, F., Holmans, P.A., Whittemore, A.S., Mowry, B.J., Olincy, A., Amin, F., Cloninger, C.R., Silverman, J.M., Buccola, N.G., Byerley, W.F., Black, D.W., Crowe, R.R., Oksenberg, J.R., Mirel, D.B., Kendler, K.S., Freedman, R., Gejman, P.V. 2009. Common variants on chromosome 6 p22.1 are associated with schizophrenia. Nature 460:753-757.

Stefansson, H., Ophoff, R.A., Steinberg, S., Andreassen, O.A., Cichon, S., Rujescu, D., Werge, T., Pietiläinen, O.P., Mors, O., Mortensen, P.B., Sigurdsson, E., Gustafsson, O., Nyegaard, M., Tuulio-Henriksson, A., Ingason, A., Hansen, T., Suvisaari, J., Lonnqvist, J., Paunio, T., Børglum, A.D., Hartmann, A., Fink-Jensen, A., Nordentoft, M., Hougaard, D., Norgaard-Pedersen, B., Böttcher. Y., Olesen, J., Breuer, R., Möller, H.J., Giegling, I., Rasmussen, H.B., Timm, S., Mattheisen, M., Bitter, I., Réthelyi, J.M., Magnusdottir, B.B., Sigmundsson, T., Olason, P., Masson. 
G., Gulcher, JR., Haraldsson, M., Fossdal, R., Thorgeirsson, TE., Thorsteinsdottir, U., Ruggeri, M., Tosato, S., Franke, B., Strengman, E., Kiemeney, LA; Genetic Risk and Outcome in Psychosis (GROUP), Melle, I., Djurovic, S., Abramova, L., Kaleda, V., Sanjuan, J., de Frutos, R., Bramon, E., Vassos, E., Fraser, G., Ettinger, U., Picchioni, M., Walker, N., Toulopoulou, T., Need, A.C., Ge, D., Yoon, J.L., Shianna, K.V., Freimer, N.B., Cantor, R.M., Murray, R., Kong, A., Golimbet, V., Carracedo, A., Arango, C., Costas, J., Jönsson, E.G., Terenius, L., Agartz, I., Petursson, H., Nöthen, M.M., Rietschel, M., Matthews, P.M., Muglia, P., Peltonen, L., St Clair, D., Goldstein, D.B., Stefansson, K., Collier, D.A. 2009. Common variants conferring risk of schizophrenia. Nature 460:744-747.
Tay, Y., Zhang, J., Thomson, A.M., Lim, B., Rigoutsos, I. 2008. MicroRNAs to Nanog, Oct4 and Sox 2 coding regions modulate embryonic stem cell differentiation. Nature. 455: 11241128.

Vasudevan, S., Tong, Y., Steitz, J.A. 2007a. AU-rich-element-mediated upregulation of translation by FXR1 and Argonaute 2. Cell. 128:11051118.

Vasudevan, S., Tong, Y., Steitz, J.A. 2007b. Switching from repression to activation: microRNAs can upregulate translation. Science. 318: 1931-1934.

Vasudevan, S., Tong, Y., Steitz, J.A. 2008. Cell-cycle control of microRNAmediated translation regulation. Cell Cycle. 7: 1545-1549.

Zoghbi, H.Y. 2005. MeCP2 dysfunction in humans and mice. J Child Neurol. 20: 736-740.

\section{How to cite this article:}

Tihomir Iliev Vachev, Nikolay Todorov Popov, Vili Krasteva 